\title{
Growth of Hierarchical CdS Nanostructures on Fluorine- Doped Tin Oxide Glass Substrate
}

Man Suk Song and Yong Kim*

Department of Physics, Dong-A University, Hadan-2-dong, Saha-gu, Busan 604-714, Korea.

E-mail:yongkim@dau.ac.kr. Telephone: 82-51-200-7276 


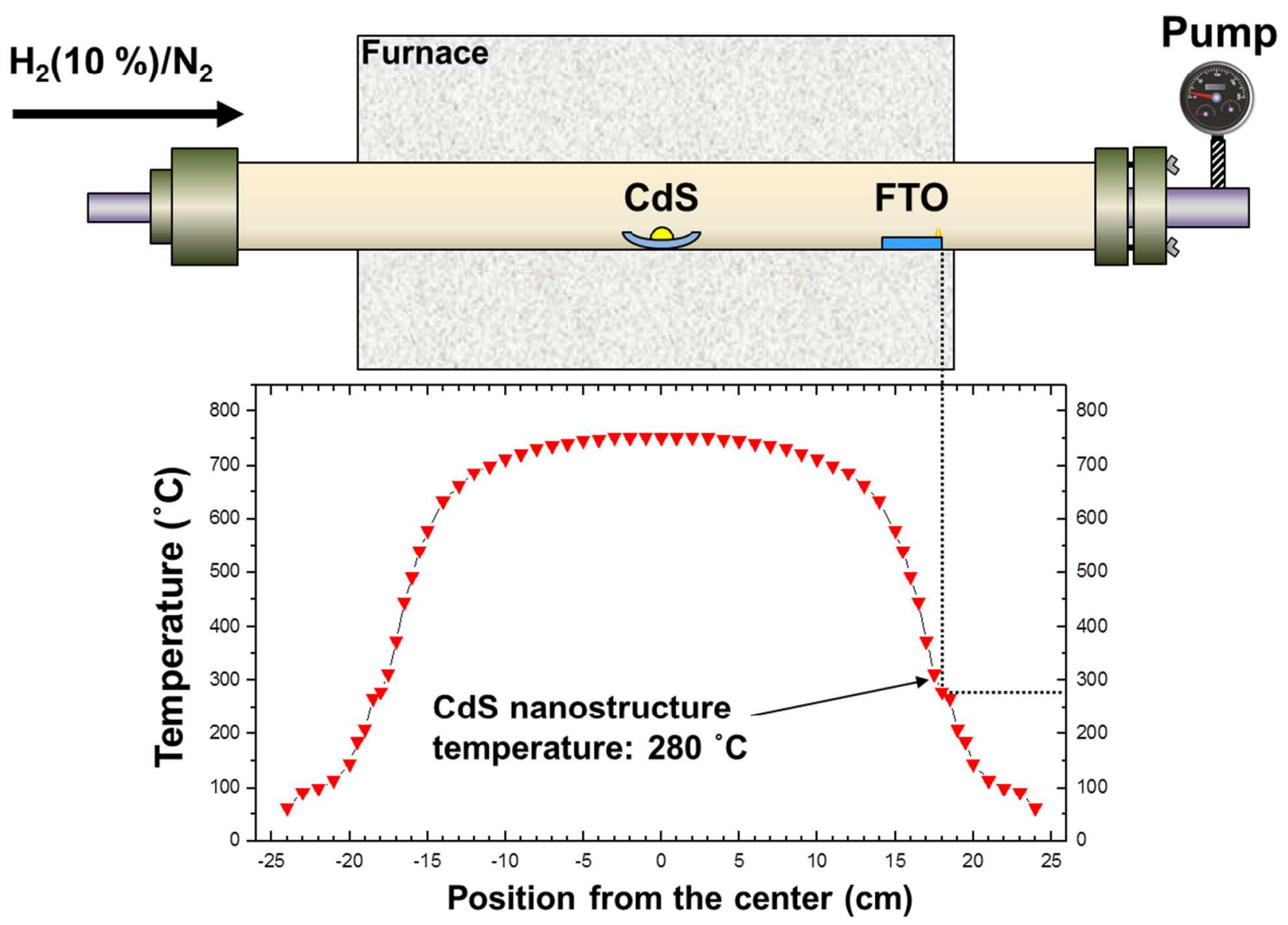

Figure S1. Physical vapor transport system and temperature profile. 


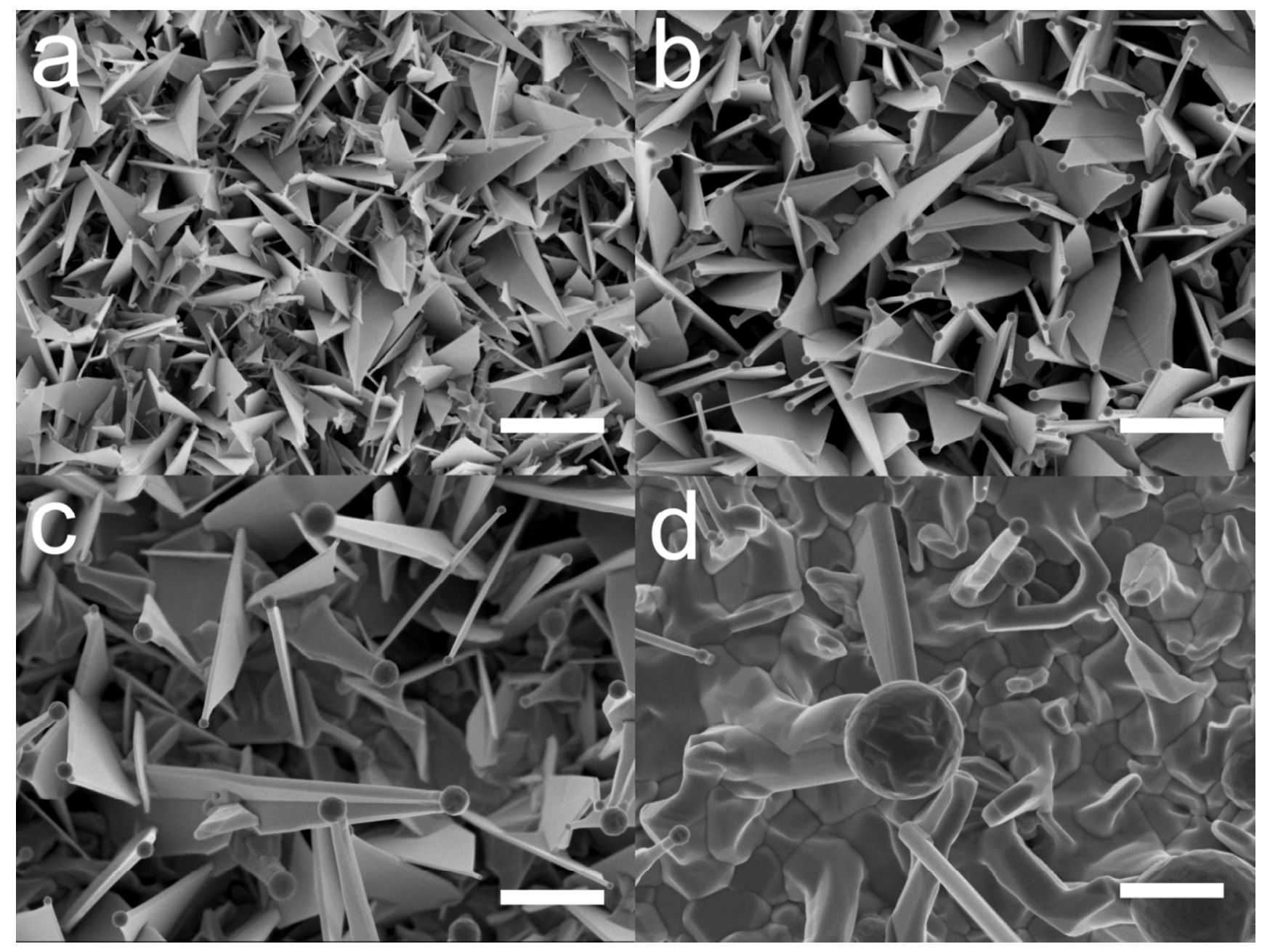

Figure S2. FESEM images of CdS nanostructure at the substrate temperatures of (a) 310, (b) 370, (c) 445, and (d) $490^{\circ} \mathrm{C}$. Scale bars are $2 \mu \mathrm{m}$. 

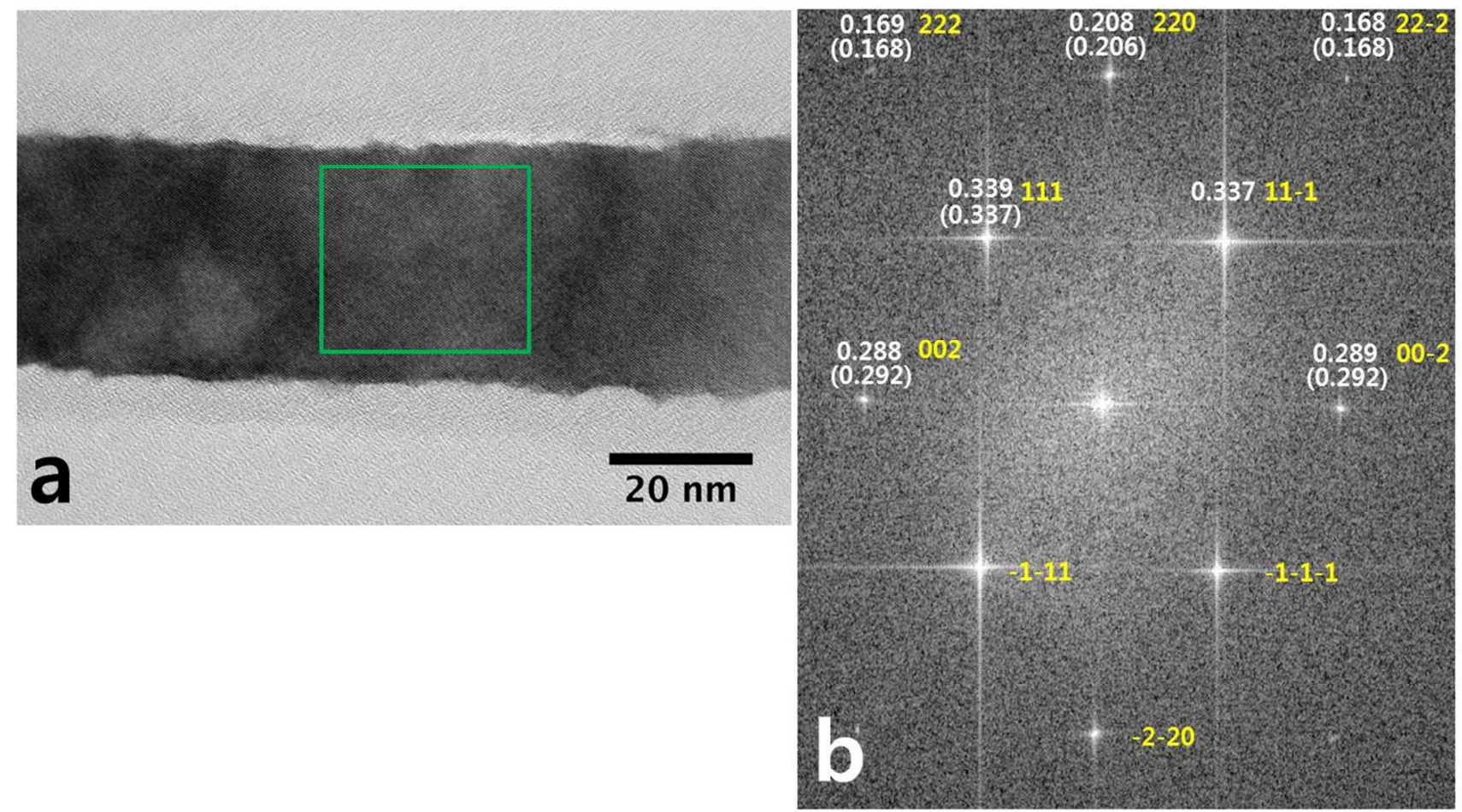

Figure S3. (a) High-Resolution TEM image of zinc blende stem. (b) A detailed FFT analysis of the green box in the ZB phase stem. The values in the parentheses in the FFT spectrum are the values from JCPDS 89-0440. 


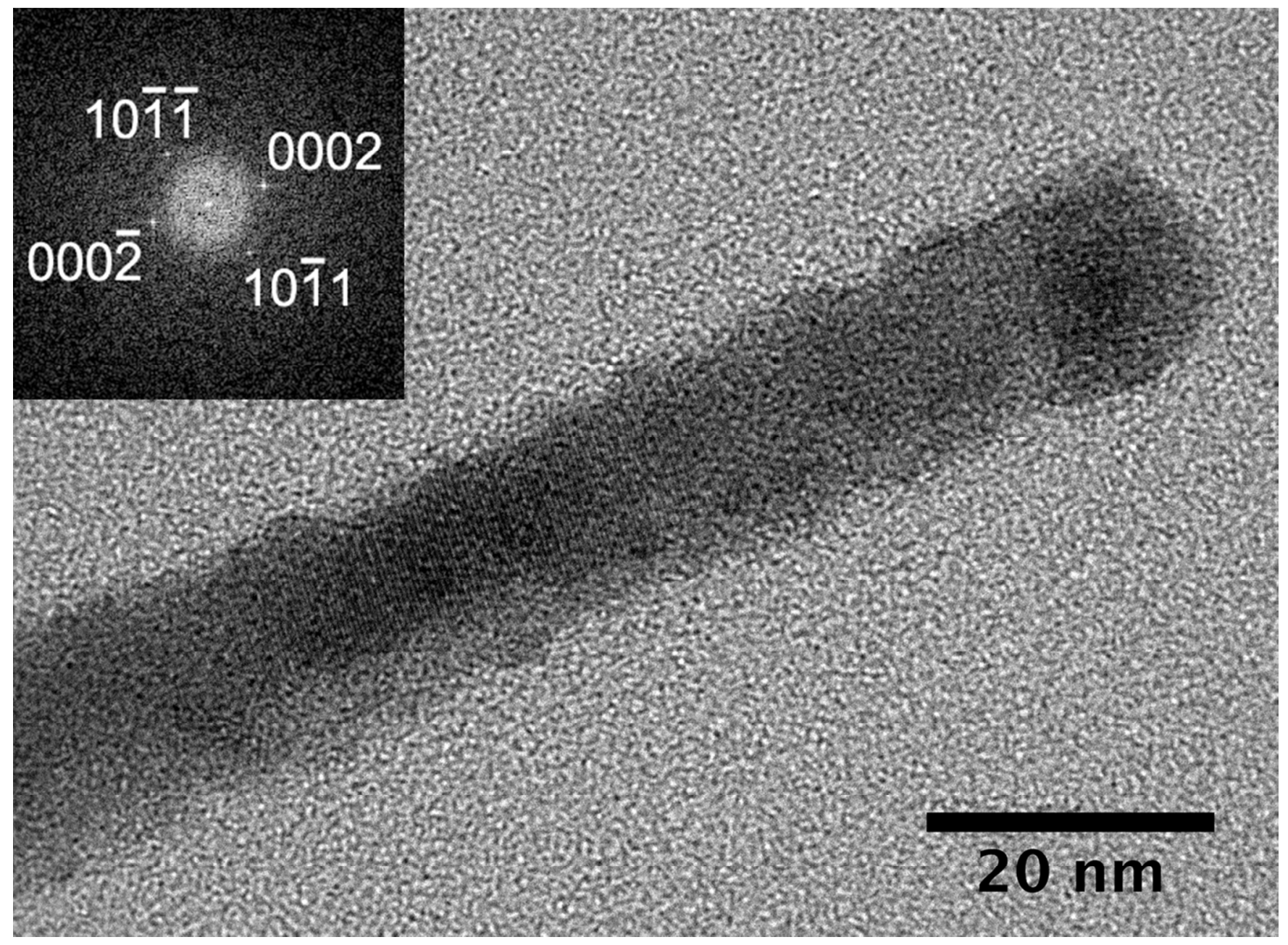

Figure S4. High-Resolution TEM image of a branch. The inset is the FFT of CdS branch. Note that the catalyst is polycrystalline. 